\title{
Análisis de los Términos Utilizados en la Literatura Científi- ca para Referirse a la Cápsula Articular y Ligamentos Arti- culares de la Articulación Temporomandibular
}

\author{
Analysis of Terms Used in the Literature to Refer to \\ Temporomandibular Joint Capsule and Joint Ligaments
}

Ramón Fuentes*; Nicolás Ernesto Ottone ${ }^{*, * *}$; Cristina Bucchi \& Mario Cantín ${ }^{* *, * * * *}$

FUENTES, R.; OTTONE, N. E.; BUCCHI, C. \& CANTÍN, M. Análisis de los términos utilizados en la literatura científica para referirse a la cápsula articular y ligamentos articulares de la articulación temporomandibular. Int. J. Morphol., 34(1):342-350, 2016.

RESUMEN: La identificación de las estructuras anatómicas con un término único, informativo y con ausencia de homónimos, entre otros principios, es la tarea primordial que lleva adelante la Federación Internacional de Programas de Terminologia Anatomica (FIPAT) perteneciente a la International Federation of Anatomical Associations (IFAA). Sin embargo, en la literatura científica, sobre todo con orientación clínica y quirúrgica, existen aún dificultades para adoptar la Terminologia Anatomica Internacional (TAI), generándose múltiples denominaciones para una misma estructura anatómica, complicando la comunicación científica. El objetivo de este trabajo fue analizar los términos utilizados en la literatura científica en relación a la cápsula articular y los ligamentos articulares de la articulación temporomandibular. Se revisaron artículos científicos y también libros de texto de anatomía general y específicos de la articulación temporomandibular. Se compararon los términos adoptados en la literatura especializada con la establecida en la TAI, identificándose los términos de la TAI más utilizados, como así también aquellos mencionados en artículos científicos y libros de texto pero no presentes en la TAI. El análisis realizado en esta investigación permitió identificar el uso de términos presentes y ausentes en la TAI, analizando su denominación, origen, inserción y función específica.

PALABRAS CLAVE: Terminologia Anatomica; Cápsula articular; Ligamentos articulares; Articulación temporomandibular.

\section{INTRODUCCIÓN}

La Terminologia Anatomica Internacional (TAI) (FICAT, 1998; FCAT, 2001) es la encargada de normalizar y estandarizar los términos que identifican a las estructuras anatómicas, estableciendo de esta manera un lenguaje común y uniforme que pueda ser utilizado por todos los morfólogos e investigadores en la enseñanza y la comunicación científica (Whitmore, 1999; Fuentes et al., 2014a, 2014b). La TAI está en constante revisión en diversos ámbitos de reunión científica, como son los Simposios Iberolatinoamericanos de Terminologia Anatomica, Embryologica e Histologica (SILAT) iniciados en 2009 y continuados hasta la actualidad (Cruz Gutiérrez et al., 2010).

En la literatura científica, la utilización de la TAI aún no se ha impuesto, y de esta manera surgen inconvenientes de denominación entre los distintos libros de anatomía, como así también en los artículos científicos publicados sobre la materia. Además, en la TAI también se encuentran ausentes algunos términos correspondientes a estructuras anatómicas cuyos hallazgos no son recientes y que son cada vez más aceptados por los investigadores y por lo tanto, necesitarían una posible incorporación a la TAI.

El objetivo de esta investigación consistió en analizar la literatura científica, libros y artículos, en relación al uso de los términos referentes a la descripción, clasificación, origen, inserción y función de la cápsula articular y los ligamentos articulares y contrastar la utilización de estas denominaciones con las establecidas por la Terminologia Anatomica Internacional. Además, se analizaron otras es-

\footnotetext{
* CICO - Centro de Investigación en Ciencias Odontológicas, Facultad de Odontología, Universidad de La Frontera, Temuco, Chile.

** Programa de Doctorado en Ciencias Morfológicas, Facultad de Medicina, Universidad de La Frontera, Temuco, Chile.

*** CIMA - Centro de Investigación en Morfología Aplicada, Facultad de Odontología, Universidad de La Frontera, Temuco, Chile.

***** Centro de Investigación en Ciencias Biomédicas, Universidad Autónoma de Chile, Temuco, Chile.
} 
tructuras no consideradas en la TAI. De esta forma, se complementan los trabajos realizados previamente con respecto a los ligamentos esfenomandibular, estilomandibular, el rafe pterigomandibular y los componentes óseos (Fuentes et al., 2014a, 2014b).

\section{MATERIAL Y MÉTODO}

Se realizó una revisión narrativa de la literatura. Se analizaron libros de ciencias morfológicas, de idiomas español, portugués e inglés y que trataban sobre la articulación temporomandibular. Se estudió específicamente lo referente a la cápsula articular, ligamento lateral, ligamento medial y otras esctructuras no presentes en la TAI, como el ligamento de Tanaka, la zona bilaminar, el ligamento posterior y los ligamentos colaterales.

Se realizó una búsqueda de artículos científicos en PubMed, Embase y SciELO. Se utilizaron las palabras clave: cápsula articular, ligamento lateral, ligamento medial, ligamento de Tanaka, ligamento posterior, ligamentos colaterales, articulación temporomandibular y Terminologia Anatomica Internacional y se combinaron con los términos boleanos AND y OR.

Se utilizó una ficha de recolección de datos, en donde se anotó la información relevante al trabajo. La extracción de los datos fue realizada en dos oportunidades por un único investigador.

Los términos utilizados en los textos revisados, fueron comparados entre sí y con Terminologia Anatomica Internacional en su versión de latín, inglés y español (año 2001) y se procedió a su análisis. En los casos de libros escritos en inglés y portugués, los términos se tradujeron al español de manera literal.

\section{RESULTADOS}

Cápsula articular (Fig. 1 y 2)
Denominación. Los términos hallados en esta revisión para denominar a la cápsula articular pueden visualizarse en la Tabla I.

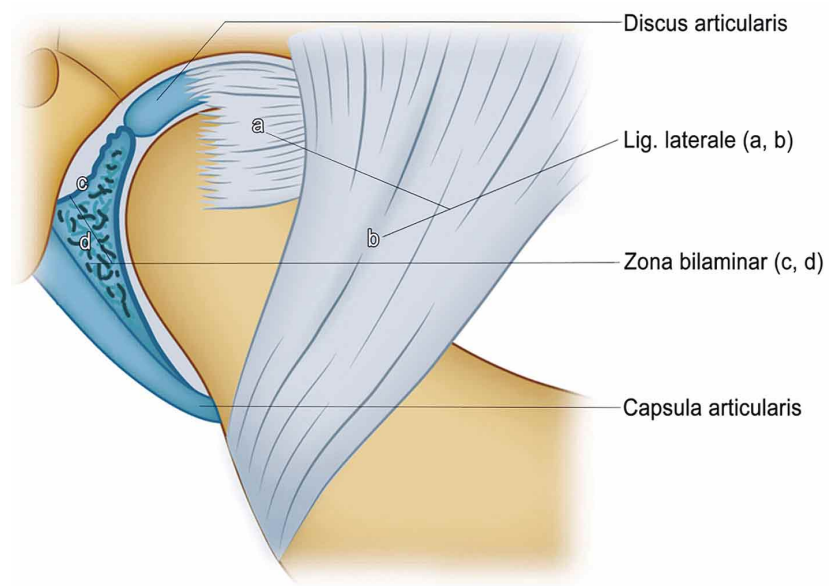

Fig. 1. Vista lateral de la articulación temporomandibular, donde se pueden apreciar el disco articular (discus articularis), el ligamento lateral (lig. laterale) con sus dos porciones, horizontal (a) y oblicua (b), la zona bilaminar, con sus fibras elásticas (c) y colágenas (d), y finalmente la cápsula articular (capsula articularis) (ver descripción detallada en el texto).

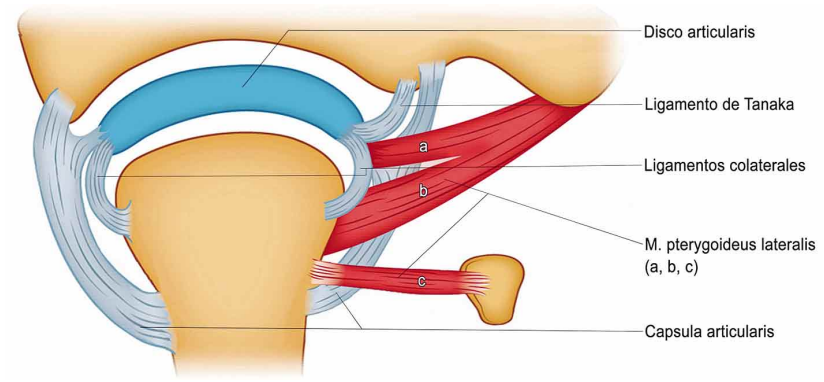

Fig. 2. Corte coronal de la articulación temporomandibular en el que se pueden apreciar el disco articular (discus articularis) y su relación con los ligamentos de Tanaka y colaterales (no descriptos en la TAI). También se puede observar la diferente disposición de la cápsula articular (capsula articularis) en relación a su inserción en el cuello del cóndilo. Y finalmente, el músculo pterigoideo lateral (M. pterygoideus lateralis), con sus dos fascículos superior (a, b) e inferior (c). El fascículo superior a su vez se divide en dos porciones, una menor grosor que se insertará en el disco articular y otra de mayor grosor que se insertará en el cuello del cóndilo (ver descripción detallada en el texto).

Tabla I. Denominación de la cápsula articular (Capsula articularis) en la literatura científica.

\begin{tabular}{ll}
\hline Cápsula articular & Spalteholz (1900); Langton \& Eggleton (1992); Mackinnon \& Morris (1993); Buman \& \\
(Término usado por TAI, 2001) & Lotzmann (2000); Feneis \& Dauber (2000); Diaz Santana (2001); Latarjet \& Ruiz Liard \\
& (2006); Schünke et al. (2010) \\
Ligamento capsular & Okeson (2003) \\
Cápsula fibrosa & Ramfjord \& Ash (1972); Helland (1980); Isberg (2003) \\
Cápsula & Alves \& Candido (2009) \\
\hline
\end{tabular}


Clasificación. Buman \& Lotzmann (2000) clasifican a la cápsula articular en lateral, medial y dorsal, y además, diferencian la porción anterior de la cápsula en anterior superior e inferior. Okeson (2003) clasifica a la cápsula articular como ligamento articular. Sin embargo, Ramfjord \& Ash (1972) e Isberg (2003) clasifican la cápsula articular como fibrosa, coincidiendo esto con la denominación otorgada. Alves \& Candido (2009), por su parte, clasifican la cápsula en dos capas, una más externa, la membrana fibrosa y otra más interna, la membrana sinovial, responsable, ésta última, de la producción del líquido sinovial.

Latarjet \& Ruiz Liard (2006), consideran que los ligamentos lateral, y también el medial, son espesamientos de la cápsula articular, a la cual refuerzan de cada lado del cóndilo.

Díaz Santana (2001) describe a la cápsula articular como más gruesa en su región superior y que de forma gradual va adelgazándose hacia la zona del cuello del cóndilo, en dirección postero inferior, extendiéndose directamente las fibras superficiales desde el hueso temporal a la mandíbula, mezclándose en el medio de este trayecto, las fibras anteriores y posteriores de la cápsula con las del disco articular. Ramfjord \& Ash, respecto a esta cuestión, consideran que la porción de la cápsula entre el disco articular y el hueso temporal es más laxa que la porción que se extiende desde el disco articular al cuello de la mandíbula.

Mackinnon \& Morris (1993) clasifican a la cápsula articular como un "cono fibroso", que rodea a la articulación, y es laxo, además de estar formado por haces de dirección vertical dispuestos en dos planos: un plano superficial, de fibras largas y gruesas, que se extienden entre ambas superficies óseas, y otro plano profundo, de fibras cortas, que se detienen en los márgenes del disco articular, permitiendo establecer un división de la articulación temporomandibular como supradiscal e infradiscal.

Origen. Langton \& Eggleton identifican el origen de la cápsula articular, a lo largo de la base de la fosa temporal, continuándose su porción medial hasta el nivel de la espina del esfenoides.

Por su parte, Mackinnon \& Morris hacen una descripción más detallada del origen de la cápsula, considerando que su circunferencia superior o base de la cápsula se inserta en los límites del área temporal: por delante, en el tubérculo articular; atrás, en el margen anterior de la fisura timpanoescamosa; por fuera, en el proceso cigomático y la raíz longitudinal del hueso cigomático, y por medial (coincidiendo con Langton \& Eggleton) en la base de la espina del esfenoides (Diaz Santana; Isberg; Latarjet \& Ruiz Liard).
Isberg también detalla que en su zona medial, la cápsula se adhiere a la sutura esfenoescamosa.

Para Okeson, el "ligamento capsular", como define a la cápsula articular, se inserta, en su origen, en el hueso temporal a lo largo de los márgenes de las superficies articulares de la fosa mandibular y el tubérculo articular. Con respecto a esto, Latarjet \& Ruiz Liard establecen que la cápsula articular se inserta en las siguientes referencias óseas: superior y posterior, en el hueso temporal, medialmente, en el labio anterior de la fisura petroescamosa, y más lateralmente, en la fisura timpanoescamosa. Superior y lateralmente, en el margen lateral de la fosa mandibular del hueso temporal.

Inserción. Mackinnon \& Morris definen una circunferencia inferior o vértice, correspondiente a la inserción distal de la cápsula, que es oblicua hacia inferior y posterior, más estrecha y se fija en el contorno de la superficie articular, excepto posteriormente, donde desciende hasta el cuello de la mandíbula, con una extensión aproximada de $5 \mathrm{~mm}$, por debajo del revestimiento fibrocartilaginoso. Así, se explica que una buena parte dorsal del cuello, quede incluida en el interior de la articulación.

Por su parte, Buman \& Lotzmann consideran que la inserción de la cápsula articular en el proceso condilar es superficial y varía según la localización en altura. La inserción de la cápsula articular en el proceso condilar ocupa una superficie bastante más grande a nivel posterior que anterior. La cápsula articular anterior se inserta de una manera más superior que la posterior, debido a la inserción del fascículo inferior del músculo pterigoideo lateral al proceso condilar.

Respecto a esto, Diaz Santana indica que posteriormente, la cápsula, también se inserta en el margen posterior de la rama de la mandíbula, justo por debajo de la zona del cuello de la mandíbula (Okeson; Latarjet \& Ruiz Liard), de modo que buena parte de éste queda incluida en la articulación; en cambio, la inserción anterior en la mandíbula se hace en la vecindad del revestimiento cartilaginoso, coincidiendo con Mackinnon \& Morris.

Langton \& Eggleton consideran que el margen anterior de la cápsula actúa como un sector de inserción miofascial para algunas de las fibras superiores del músculo pterigoideo lateral. Diaz Santana indica que una pequeña zona anterior y medial de la articulación no presenta cápsula articular, y se corresponde a su vez con el fascículo superior del músculo pterigoideo lateral, que se inserta directamente en el disco articular, en su región anterior, estableciéndose una relación de continuidad de las fibras 
musculares con el disco articular. También Mackinnon \& Morris coinciden con esto, indicando la ausencia de cápsula articular como entidad anatómica en la cara antero medial de la articulación, donde se da la fusión de algunas fibras del fascículo superior del músculo pterigoideo lateral con el disco articular. En relación a esto, Naidoo \& Juniper (1997) analizaron 40 muestras, tanto macroscópicas como histológicas, y encontraron que el $29,5 \%$ de las fibras del fascículo superior del músculo pterigoideo lateral se insertan en el tercio medial del disco articular. Por su parte, Bittar et al. (1994) describieron este hallazgo, en piezas de histología, en solo el 2,4\% al 6,3\%. Finalmente, Pompei Filho et al. (2010) al analizar estudios de resonancia magnética de 103 individuos, encontraron la inserción de las fibras del fascículo superior del músculo pterigoideo lateral en el disco articular en 69,8-70,7\% de las muestras de mujeres $(n=71)$ y en el 68,9-74,2\% de las muestras de hombres $(\mathrm{n}=32)$. Por su parte, Anatonopoulou et al., (2013) encontraron 3 formas distintas de inserción del músculo pterigoideo lateral, siendo la más frecuente, $55,5 \%$, a nivel del proceso condilar, en la fóvea pterigoidea. En el 27,8 $\%$ de los casos se insertaba directamente en el cóndilo de la mandíbula, y en solo el 16,7 \% a nivel del "complejo cápsula-disco articular".

Función. Según Okeson la cápsula articular actúa oponiendo resistencia ante cualquier fuerza interna, externa o inferior que provoque separación o luxación de las superficies articulares. Una función importante de la cápsula articular es envolver la articulación, además de producir y retener el líquido sinovial.

\section{Ligamento lateral (Fig. 1)}

Denominación. La denominación del ligamento lateral (Ligamentum laterale) en la literatura científica se detalla en la Tabla II.

Clasificación. Ramfjord \& Ash clasifican al ligamento lateral como temporomaxilar externo. Con esta modalidad coinciden Mackinnon \& Morris, pero clasificándolo directamente como ligamento lateral externo.
Para Langton \& Eggleton, el ligamento lateral presentaría dos fascículos de origen. Buman \& Lotzmann lo clasifican formado por dos porciones: una porción profunda, con un recorrido horizontal y una porción superficial, con un recorrido oblicuo. Por su parte, Diaz Santana considera que la parte lateral de la cápsula articular, que es más gruesa que el resto, es casi la única que merece el nombre de ligamento temporomandibular o ligamento lateral (ligamentum laterale). Este autor lo clasifica con forma de abanico, con su parte ancha en la zona del arco cigomático, extendiéndose más allá del tubérculo articular, y su parte más estrecha correspondiente a la porción que se inserta en el cuello del cóndilo.

En este sentido, Okeson clasifica al ligamento lateral en dos partes: una porción oblicua externa y otra horizontal interna. Latarjet \& Ruiz Liard clasifican al ligamento lateral como uno de los ligamentos intrínsecos, junto al ligamento medial, identificándolos como espesamientos de la cápsula, a la que refuerzan de cada lado del cóndilo.

Finalmente, y solo descripto por Dufourmentel (a través de Mackinnon \& Morris), este autor reconoce dos contingentes de fibras en el ligamento lateral: uno posterior, corto y fuerte, extendido del tubérculo cigomático al cuello del cóndilo y que llama cuerda cigomatomaxilar, y otro anterior, más ancho y de menor espesor, que se extiende desde el margen medial del arco cigomático a la cara lateral de la rama, denominado la bandeleta cigomatomaxilar.

Origen. Ramfjord \& Ash consideran que el origen del ligamento lateral corresponde a la superficie externa de un proceso cigomático. Okeson coincide con esto, pero incluyendo también al tubérculo articular. Langton \& Eggleton determina los mismos orígenes para el ligamento lateral, pero dividiéndolo en porciones anterior y posterior.

Mackinnon \& Morris describen un ligamento lateral externo (ligamento lateral según TAI) se origina a través de haces de refuerzo de la cápsula que se insertan superiormente en el proceso cigomático y el tubérculo articular.

Tabla II. Denominación del ligamento lateral (Ligamentum laterale) en la literatura científica.

\begin{tabular}{ll}
\hline $\begin{array}{l}\text { Ligamento lateral } \\
\text { (Término usado por TAI, 2001) }\end{array}$ & $\begin{array}{l}\text { Langton \& Eggleton (1992); Sato et al. (1996); Buman \& Lotzmann (2000); Feneis \& } \\
\text { Dauber (2000); Diaz Santana (2001); Isberg (2003); Okeson (2003); Latarjet \& Ruiz Liard } \\
\text { (2006); Alves \& Candido (2009); Schünke } \text { et al. (2010); Cuccia } \text { et al. (2011) }\end{array}$ \\
& $\begin{array}{l}\text { Spalteholz (1900); Helland (1980); Langton \& Eggleton (1992); Feneis \& Dauber (2000); } \\
\text { Ligamento temporomandibular }\end{array}$ \\
& $\begin{array}{l}\text { Buman \& Lotzmann (2000); Diaz Santana (2001); Isberg (2003); Okeson (2003); Alves \& } \\
\text { Candido (2009); Cuccia et al. (2011) }\end{array}$ \\
Ligamento temporomaxilar externo & Ramfjord \& Ash (1972) \\
Ligamento lateral externo & Mackinnon \& Morris (1993) \\
\hline
\end{tabular}


Por su parte, Diaz Santana considera que se origina, desde su porción más ancha, en la zona del arco cigomático, extendiéndose más allá del tubérculo articular. Con esto concuerdan Alves \& Candido, pero sin hacer referencia al tamaño del ligamento.

Inserción. Todos los autores coinciden en identificar como zona de inserción del ligamento lateral al cuello de la mandíbula, sin llegar a alcanzar el margen posterior de la rama de la mandíbula (Ramfjord \& Ash; Langton \& Eggleton; Mackinnon \& Morris; Diaz Santana; Okeson; Alves \& Candido).

Hay diferencias específicas que podemos describir a continuación. Langton \& Eggleton consideran que las fibras de ligamento lateral se extienden posteriormente y en forma oblicua para insertarse en la superficie lateral y posterior del cuello de la mandíbula. La porción posterior se va a insertar en la mandíbula junto a la porción posterior de la cápsula. Respecto a esto, Mackinnon \& Morris establecen que las fibras anteriores del ligamento lateral externo son oblicuas hacia abajo y atrás (coincidiendo con Langton \& Eggleton), pero agregan que las fibras posteriores del ligamento son verticales, insertándose reunidas a las anteriores en la parte posteroinferior del cuello, a $10 \mathrm{o} 12 \mathrm{~mm}$ por debajo de la interlínea articular. Diaz Santana determina que es la porción más estrecha del ligamento lateral la que se inserta en el cuello del cóndilo.

Por su parte, Okeson (2003) considera también (al igual que Langton \& Eggleton y Mackinnon \& Morris) que la porción externa del ligamento temporomandibular, desde su origen presenta dirección posteroinferior hasta su inserción en la superficie externa del cuello del cóndilo. Por su parte, la porción horizontal interna, se extiende en dirección posterior y horizontal, hasta insertarse en el polo externo del cóndilo de la mandíbula y la parte posterior del disco articular.

Función. Ramfjord \& Ash consideran al ligamento temporomaxilar (ligamento lateral según TAI) como el más directamente relacionado con la articulación, residiendo su importancia en la limitación de los movimientos de la mandíbula. Lo dividen en interno y externo, considerando que intervienen en forma importante en la limitación de los movimientos retrusivos de la mandíbula (porción horizontal del ligamento, según Buman \& Lotzmann; Alves \& Candido). La cápsula articular y algunas porciones del ligamento lateral posiblemente sean de importancia para marcar el límite de los movimientos laterales extremos en la abertura forzada (Diaz Santana). Para Okeson la porción oblicua del ligamento TM influye en el movimiento de apertura normal de la mandíbula. Latarjet \& Ruiz Liard reducen la función de los ligamentos a simples espesamientos de la cápsula a la que refuerzan de cada lado del proceso condilar.

\section{Ligamento medial}

Respecto a este ligamento hay poca información. Es identificado como ligamento por Ramfjord \& Ash, Mackinnon \& Morris, Feneis \& Dauber, FCAT, FICAT, Isberg, Okeson y Latarjet \& Ruiz Liard. Su denominación en la literatura científica se detalla en la Tabla III.

Tanto Feneis \& Dauber (2000) como Latarjet \& Ruiz Liard lo consideran, junto al ligamento lateral, un refuerzo y espesamiento de la cápsula, en este caso de la pared medial de la misma.

Martinez Ross (1985) y Mackinnon \& Morris indican que el ligamento lateral interno (ligamento medial) se desprende de la base de la espina del esfenoides y desciende oblicuamente para insertarse en la parte postero medial del cuello, a 10 o $15 \mathrm{~mm}$ por debajo de la línea articular .

\section{Ligamentos colaterales (Fig. 2)}

Langton \& Eggleton describen que la cápsula "fibrosa" se engrosa lateralmente por los ligamentos temporomadibular y el ligamento colateral lateral, pero que por microdisección, se detecta la individualidad de estos elementos entre sí. Esto también es considerado por Ramfjord \& Ash. Sin embargo, no existen términos en la TAI para denominar a los ligamentos colaterales.

Para Okeson los ligamentos colaterales fijan los margens lateral y medial del disco articular a los polos del cóndilo. Establece que también se los denomina ligamentos discales, y son dos: 1) el ligamento discal medial/interno y 2) el ligamento discal lateral/externo. Los ligamentos colaterales/discales son ligamentos verdaderos, que están formados por fibras de tejido conjuntivo colágeno y, de esta manera, no son distensibles.

Según Langton \& Eggleton, el disco articular está adherido al cóndilo de la mandíbula a través de dos ligamentos, ligamentos colaterales medial y lateral. Indican que no son colaterales entre sí en el mismo plano y que no son del todo consistentes. El ligamento colateral lateral es más amplio y delgado, con muchas variaciones. Por su parte, el ligamento colateral medial, se inserta más inferiormente en el cuello de la mandíbula, en comparación a su homólogo lateral. Este ligamento es más grueso y, en relación al ángulo rotacional de la cabeza del cóndilo, se dispone en un plano un poco más posterior que el ligamento colateral lateral. Este ligamento se encuentra más tenso y ofrece una menor 
FUENTES, R.; OTTONE, N. E.; BUCCHI, C. \& CANTÍN, M. Análisis de los términos utilizados en la literatura científica para referirse a la cápsula articular y ligamentos articulares de la articulación temporomandibular. Int. J. Morphol., 34(1):342-350, 2016

libertad de movimiento Además, fibras del músculo pterigoideo lateral, al insertarse en el cuello del cóndilo mandibular, se entremezclan con la cápsula articular y posiblemente con el ligamento colateral medial y el disco articular.

Para Okeson, actúan limitando el movimiento de alejamiento del disco respecto del cóndilo de la mandíbula. Permiten que el disco se mueva pasivamente con el cóndilo cuando éste se desliza hacia anterior y hacia posterior. También Cuccia et al. (2011) identifican a los ligamentos colaterales lateral y medial. La TAI no tiene términos para denominar a estos ligamentos.

\section{Ligamento de Tanaka (Fig. 2)}

Langton \& Eggleton describen el ligamento de Tanaka, originalmente descubierto por Terry Tanaka a comienzos de los años 80 del siglo pasado. Este ligamento se extiende desde el disco articular, medialmente y algo anterior, para insertarse en la zona inferomedial de la fosa mandibular. Fue considerado originalmente como un fascículo medial proveniente del ligamento colateral medial, pero puede individualizarse del ligamento colateral medial y ser identificado. Este ligamento reforzaría al disco articular medialmente, contribuyendo a formar un arco medial de refuerzo, en torno a la rotación que sufre el disco durante el ciclo de apertura y de cierre.

Buman \& Lotzmann también consideran que el ligamento de Tanaka es un refuerzo alargado de la cápsula medial semejante al ligamento lateral. Cuccia et al. también lo identifican. La TAI no tiene un término para denominar a este ligamento.

\section{Zona bilaminar o Ligamento posterior (Fig. 1 y 2)}

En la región posterior de la articulación temporomandibular, pueden encontrarse la "zona bilaminar" o ligamento posterior, que se adhiere al margen posterior del disco articular, y se extiende posteriormente para fusionarse con la cápsula articular. La zona bilaminar o ligamento posterior está conformado por fibras elásticas y fibras colágenas (Siéssere et al., 2004; Taitson et al., 2010). Las fibras elásticas se disponen superiormente, extendidas desde la pared posterior de la fosa mandibular al margen poste- rior del disco articular, mientras que las fibras colágenas, de disposición inferior, se extienden del margen posterior del disco articular para insertarse en el cuello de la mandíbula (Buman \& Lotzmann; Benigno et al., 2001).

Esta zona es considerada como una especie de "almohadilla" de tejido areolar laxo, conformado por fibras colágenas, en una red de fibras elásticas (Pertes \& Gross, 2005).

Es designado como zona bilaminar por diversos autores (Parsons \& Boucher, 1966; Hall et al., 1984; van der Kuijl et al., 1990; Hollender et al., 1998; Zhang et al., 2006; Leonardi et al., 2012). Benigno et al., también identifican la existencia de la zona bilaminar, a través de estudios con microscopía de luz y escaneo electrónico, evidenciando la existencia de las fibras colágenas y las fibras elásticas, constituyentes de esta zona bilaminar o ligamento posterior.

Por su parte, Mackinnon \& Morris definen a esta estructura como ligamento posterior, representado por fibras elásticas no bien diferenciadas que se extienden desde la fisura timpanoescamosa al cuello del cóndilo y el margen posterior del disco articular. Las fibras profundas de este ligamento, limitarían la excursión del cóndilo y del disco articular en la propulsión del maxilar, transformándose en elementos activos cuando se ejecuta el movimiento antagónico.

Pertes \& Gross, también denominan a esta estructura como ligamento posterior, y lo ubican ocupando un espacio entre el disco articular y la pared posterior de la cápsula articular. La zona bilaminar se encuentra altamente vascularizada e inervada (Siéssere et al.; Pertes \& Gross; Taitson et al.). La TAI no tiene un término para denominar a este ligamento.

\section{DISCUSIÓN}

En esta discusión nos centraremos en las discrepancias existentes al momento de denominar las estructuras anatómicas aquí analizadas. Además, también destacaremos la ausencia de estructuras anatómicas relacionadas a la ATM en la TAI, que si son regularmente descritas en artículos

Tabla III. Denominación del ligamento medial (Ligamentum mediale) en la literatura científica.

\begin{tabular}{ll}
\hline Ligamento medial & Feneis \& Dauber (2000); Isberg (2003); Okeson (2003); Latarjet \& Ruiz Liard (2006); \\
(Término usado por TAI, 2001) & Cuccia et al. (2011) \\
Ligamento temporomaxilar interno & Ramfjord \& Ash (1972) \\
Ligamento lateral interno & Mackinnon \& Morris (1993) \\
\hline
\end{tabular}


científicos y libros relacionados con esta temática. Las distintas descripciones en relación al origen, inserción y función de estas estructuras fueron debidamente analizadas en la sección de resultados.

En relación a las estructuras analizadas en nuestro trabajo, veremos que existe, entre la mayoría de los autores consultados, una misma manera de denominarlas, de acuerdo a lo establecido, en la actualidad, por la TAI. Sin embargo, también existen autores que denominan en forma distinta a estas mismas estructuras, estableciendo discrepancias al momento de definir estas estructuras anatómicas, ya sea en el ámbito académico como en el clínico.

En la TAI no está identificado dentro de la sección otorgada a "articulación temporomandibular" la estructura anatómica "cápsula articular". Si lo está, en forma general, al describir al inicio de la TAI las distintas estructuras correspondientes a una articulación. Si consideramos que la TAI describe a la cápsula articular (proveniente del latín capsula articularis), veremos que la mayoría de los autores consultados respetan el uso de la TAI para definir a este elemento anatómico (Langton \& Eggleton; Mackinnon \& Morris; Buman \& Lotzmann; Diaz Santana; Latarjet \& Ruiz Liard; Schünke et al., 2010). Por su parte, Feneis \& Dauber no mencionan la presencia de la cápsula articular como tal, pero si la nombran al identificar a los ligamentos lateral y medial, a los que define como refuerzos de las paredes de la cápsula. En este sentido coinciden Latarjet \& Ruiz Liard.

Los autores que difieren de la TAI son Okeson, Isberg y Alves \& Candido, quienes la denominan ligamento capsular, cápsula fibrosa y cápsula, respectivamente. Tampoco Ramfjord \& Ash y Helland (1980) coinciden con la TAI. Estos la denominan cápsula fibrosa.

Con respecto al ligamento lateral, como es denominado en la TAI, en este caso coinciden con esta denominación (Langton \& Eggleton; Sato et al., 1996; Buman \& Lotzmann; Feneis \& Dauber; Diaz Santana; Isberg; Okeson; Latarjet \& Ruiz Liard; Alves \& Candido; Schünke et al.). Sin embargo, está muy extendido, sobre todo en artículos científicos y libros de especialidad en áreas clínicas y quirúrgicas de la ATM, a definir al ligamento lateral como ligamento temporomandibular (Helland; Langton \& Eggleton; Feneis \& Dauber; Buman \& Lotzmann; Diaz Santana; Isberg; Okeson; Alves \& Candido). Ya Spalteholz, en su atlas de 1900, lo definía Ligamentum temporomandibulare.

Por su parte, Ramfjord \& Ash, muchos años antes de la publicación de la actual TAI, lo definen como liga- mento temporomaxilar externo. Con esto coinciden Mackinnon \& Morris, quienes lo llaman ligamento lateral externo.

En referencia al ligamento medial, este es poco identificado por los autores, existiendo escasa información del mismo. Los pocos autores que lo nombran, además de la TAI, son Feneis \& Dauber, Isberg, Okeson y Latarjet \& Ruiz Liard. Por su parte, Ramfjord \& Ash y Mackinnon \& Morris, lo identifican como ligamento temporomaxilar interno y lateral interno, respectivamente.

Con respecto a la zona bilaminar, esta es identificada por una variedad de autores (Parsons \& Boucher; Hall et al.; van der Kuijl et al.; Hollender et al.; Benigno et al.; Zhang et al.; Leonardi et al.) quienes la ubican en el área retrodiscal, uniendo el disco articular a la cápsula. A su vez, Mackinnon \& Morris y Pertes \& Gross, describen un ligamento posterior, que participaría como uno de los elementos que limita los movimientos de protrusión de la mandíbula. De acuerdo a lo expuesto, y en el caso particular de la zona bilaminar o ligamento posterior, sería adecuada su incorporación a la TAI con la denominación "ligamento posterior". Además, también se podrían incluir, dependiendo del término "ligamento posterior", los términos fibras elásticas y fibras colágenas.

A continuación, haremos referencia a estructuras ligamentarias que se encuentran en la ATM, y que son descriptas en artículos científicos y libros, pero que no están incluidas en la TAI, estas son: ligamento colateral lateral y medial y el ligamento de Tanaka.

Los ligamentos colaterales son identificados por Langton \& Eggleton y Okeson. Este último autor también los define como ligamentos discales. Estos autores los consideran como verdaderos engrosamientos o refuerzos de la cápsula articular.

Por su parte, al ligamento de Tanaka se lo describe formando parte de la ATM junto a los ligamentos colaterales, presentando una ubicación superior al ligamento colateral medial.

Sin embargo, al igual que los ligamentos colaterales, el ligamento de Tanaka no está lo suficientemente investigado, desde el punto de su estructura morfológica, es decir, desde la disección cadavérica, como para precisar y asegurar la existencia de estas estructuras, debido a que su hallazgo en la cirugía es remota por la dificultad de su visualización. Junto a estos elementos anatómicos podría incorporarse también al ligamento medial, cuya existencia anatómica real tampoco está del todo definida, existiendo solo descripciones teóricas de su presencia. 
A partir de todo lo expuesto, consideramos de gran importancia el establecimiento de un idioma común a todos los investigadores y profesionales que trabajan en la ATM, en relación a sus estructuras anatómicas, por ello es muy importante la clasificación establecida por la TAI. Pero también entendemos que la misma debería ser ampliada con las estructuras que no se encuentran incluidas (ligamentos colaterales, ligamento de Tanaka) o que se encuentran en la TAI, pero que cuentan con escasa evidencia de individualidad morfológica, más allá de la descriptiva, la cual además es escasa (ligamento medial).

Se hace necesario aclarar que el cóndilo de la mandíbula es empleado erróneamente por numerosos autores, como puede ser observado en este trabajo. Lo correcto, de acuerdo a Terminologia Anatomica, sería proceso condilar, el cual comprende el cóndilo de la mandíbula, el cuello de la mandíbula y fosa pterigoidea.

Finalmente, consideramos que para lograr ampliar la TAI, son necesarios estudios futuros que permitan, a partir de tareas de disección cadavérica y posteriores análisis morfológicos, buscar estos elementos anatómicos para definir en forma precisa su existencia, y posteriormente su ubicación, origen, inserción y relaciones.

FUENTES, R.; OTTONE, N. E.; BUCCHI, C. \& CANTÍN, M. Analysis of terms used in the literature to refer to temporomandibular joint capsule and joint ligaments. Int. J. Morphol., 34(1):342-350, 2016.

SUMMARY: The identification of anatomical structures with a single, informative term and absence of homonyms, among other principles, the primary task is being conducted by the International Federation of programs Anatomical Terminology (FIPAT) belonging to the International Federation of Anatomical Associations (IFAA). However, in the literature, particularly with clinical and surgical direction, there is still difficulty in adopting the Terminologia Anatomica Internacional (TAI), generating multiple names for the same anatomical structure, complicating scientific communication. The aim of this study was to analyze the terms used in the literature in relation to the joint capsule and joint ligaments of the temporomandibular joint. Scientific papers and textbooks on general and specific anatomy, of the temporomandibular joint is reviewed. We compared the terms adopted with TAI literature specialized terms, identifying the TAI terms most used, as well as those mentioned in scientific and textbook literature, but not present in the TAI. The analysis in this study identified the use of terms present and absent in TAI, analyzing their name, origin, insertion and specific function.

KEYWORDS: Terminologia Anatomica; Joint capsule; Articular ligaments; Temporomandibular joint.

\section{REFERENCIAS BIBLIOGRÁFICAS}

Alves, N. \& Candido, P. L. Anatomia para o Curso de Odontología Geral e Especifica. São Paulo, Livraria Santos Editora, 2009.

Antonopoulou, M.; Iatrou, I.; Paraschos, A. \& Anagnostopoulou, S. Variations of the attachment of the superior head of human lateral pterygoid muscle. J. Craniomaxillofac. Surg., 41(6):e91-7, 2013.

Benigno, M. I.; Azeredo, R. A.; Lemos, J. L.; König Júnior, B. \& Liberti, E. A. The structure of the bilaminar zone in the human temporomandibular joint: a light and scanning electron microscopy study in young and elderly subjects. J. Oral Rehabil., 28(2):113-9, 2001.

Bittar, G. T.; Bibb, C. A. \& Pullinger, A. G. Histologic characteristics of the lateral pterygoid muscle insertion to the temporomandibular joint. J. Orofac. Pain, 8(3):243-9, 1994.

Buman, A. \& Lotzmann, U. Atlas de Diagnóstico funcional y principios terapéuticos en odontología. Barcelona, Masson, 2000 .

Comité Federal sobre Terminología Anatómica (FCAT). Terminología Anatómica. Terminología Anatómica Internacional. Madrid, Ed. Médica Panamericana, 2001.

Cruz Gutiérrez, R.; Rodríguez Torres, A.; Prates, J. C.; Losardo, R. J. \& Valverde Barbato de Prates, N. Simposio Ibero-latinoamericano de Terminología Morfológica. Características generales. Int. J. Morphol., 28(2):643-6, 2010.

Cuccia, A. M.; Caradonna, C. \& Caradonna, D. Manual therapy of the mandibular accessory ligaments for the management of temporomandibular joint disorders. J. Am. Osteopath. Assoc., 111(2):102-12, 2011.

Diaz Santana, H. Anatomía de la cabeza: con enfoque odontoestomatológico. $3^{\mathrm{a}}$ ed. Madrid, Médica Panamericana, 2001.

Federative International Committee on Anatomical Terminologies (FICAT). Terminologia Anatomica. International Anatomical Terminology. New York, Thieme, 1998.

Feneis, H. \& Dauber, W. Pocket Atlas of Human Anatomy. Based on the International Nomenclature. $4^{\text {th }}$ ed. Stuttgart, Georg Thieme Verlag, 2000.

Fuentes, R.; Ottone, N.; Cantin, M. \& Bucchi, C. Analysis of Terms Used in Scientific Literature Referred to Extracapsular Ligaments of the Temporomandibular Joint. Part II: Ligamentum stylomandibulare and Raphe pterymandibulare. Int. J. Morphol., 32(4):1289-95, 2014a. 
FUENTES, R.; OTTONE, N. E.; BUCCHI, C. \& CANTÍN, M. Análisis de los términos utilizados en la literatura científica para referirse a la cápsula articular y ligamentos articulares de la articulación temporomandibular. Int. J. Morphol., 34(1):342-350, 2016.

Fuentes, R.; Cantin, M. \& Bucchi, C. A study of terms used in scientific literature to refer to the extracapsular ligaments of the temporomandibular joint. Part I: Ligamentum sphenomandibulare. Int. J. Morphol., 32(3):981-6, 2014 b.

Hall, M. B.; Brown, R. W. \& Baughman, R. A. Histologic appearance of the bilaminar zone in internal derangement of the temporomandibular joint. Oral Surg. Oral Med. Oral Pathol., 58(4):375-81, 1984.

Helland, M. M. Anatomy and function of the temporomandibular joint. J. Orthop. Sports Phys. Ther., 1(3):145-52, 1980.

Hollender, L.; Barclay, P.; Maravilla, K. \& Terry, V. The depiction of the bilaminar zone of the temporomandibular joint by magnetic resonance imaging. Dentomaxillofac. Radiol., 27(1):45-7, 1998.

Isberg, A. Disfunción de la articulación temporomandibular. Una guía práctica. San Pablo, Artes Médicas, 2003.

Langton, D. P. \& Eggleton, T. M. Functional anatomy of the temporomandibular joint complex. Santiago de Chile, Inforc Publications, 1992.

Latarjet, M. \& Ruiz Liard, A. Anatomía Humana. $4^{\mathrm{a}}$ ed. Tomo 2. Buenos Aires, Médica Panamericana, 2006.

Leonardi, R.; Rusu, M. C.; Loreto, F.; Loreto, C. \& Musumeci, G. Immunolocalization and expression of lubricin in the bilaminar zone of the human temporomandibular joint disc. Acta Histochem., 114(1):1-5, 2012.

Mackinnon, P. C. B. \& Morris, J. F. Oxford Anatomía Funcional. Cabeza y Cuello. Volumen III. Bogotá, Médica Panamericana, 1993.

Martinez Ross, E. Oclusión orgánica. México D.F., Salvat, 1985. pp.59-60.

Naidoo, L. C. \& Juniper, R. P. Morphometric analysis of the insertion of the upper head of the lateral pterygoid muscle. Oral Surg. Oral Med. Oral Pathol. Oral Radiol. Endod., 83(4):441-6, 1997.

Okeson, J. P. Tratamiento de oclusión y afecciones temporomandibulares. $5^{\text {a }}$ ed. Madrid, Elsevier Mosby, 2003.

Parsons, M. T. \& Boucher, L. J. The bilaminar zone of the meniscus. J. Dent. Res., 45(1):59-61, 1966.

Pertes, R. \& Gross, S. Tratamento Clínico das disfunçoes Temporomandibulares e da dor Orofacial. São Paulo, Quintessence, 2005.

Pompei Filho, H.; Suazo Galdames, I. \& Guimarães, A. S. Superior head of the lateral pterygoid muscle inserting in asymptomatic temporomandibular joints. Int. J. Odontostomat., 4(1):19-22, 2010.
Ramfjord, S. P. \& Ash, M. M. Jr. Occlusion. $2^{\mathrm{a}}$ ed. México D. F., Nueva Editorial Interamericana, 1972.

Sato, I.; Shindo, K.; Ezure, H. \& Shimada, K. Morphology of the lateral ligament in the human temporomandibular joint. Oral Surg. Oral Med. Oral Pathol. Oral Radiol. Endod., 81(2):1516, 1996.

Schünke, M.; Schulte, E. \& Schumacher, U. Prometheus: texto y atlas de anatomía. $2^{\mathrm{a}}$ ed. Madrid, Médica Panamericana, 2010.

Siéssere, S.; Vitti, M.; Souza, L. G.; Semprini, M. \& Regalo, S. C. Bilaminar zone: anatomical aspects, irrigation and inervation. Braz. J. Morphol. Sci., 21(4):217-20, 2004.

Spalteholz, W. Hand Atlas of Human Anatomy. Vol. I. Bones, Joints, Ligaments. Leipzig, S. Hirzel., 1900. pp.152-5.

Taitson, P. F.; Coelho Filho, D.; Siqueira Pêgo, R. \& Fonseca Ferreira, Y. Zona bilaminar: aspectos anatômicos, histológicos e funcionais. Arq. Bras. Odontol., 6(2):71-7, 2010.

van der Kuijl, B.; Vencken, L. M.; de Bont, L. G. \& Boering, G. Temporomandibular joint computed tomography: development of a direct sagittal technique. J. Prosthet. Dent., 64(6):709-15, 1990.

Whitmore, I. Terminologia Anatomica: new terminology for the new anatomist. Anat. Rec., 257(2):50-3, 1999.

Zhang, Y. K.; Gu, Z. Y. \& Hu, Q. G. Experimental study of the mechanism of chondroid metaplasia of the bilaminar zone following anterior disc displacement of temporomandibular joint. Shanghai Kou Qiang Yi Xue, 15(1):48-51, 2006.

\section{Dirección para Correspondencia: \\ Dr. Ramón Fuentes}

$\mathrm{CICO}$ - Centro de Investigación en Ciencias Odontológicas

Facultad de Odontología

Universidad de La Frontera

Manuel Montt 112, Casilla 54-D

Temuco

CHILE

\section{Email: ramon.fuentes@ufrontera.cl}

Recibido : 29-09-2015

Aceptado: 05-11-2015 